\title{
Development of Web Services for WLAN-based Indoor Positioning and Floor Map Repositories
}

\author{
Jaegeol Yim \\ Dept. of Computer Engineering Dongguk University at Gyeongju, \\ Gyeongju, Gyeongbuk, Korea \\ yim@dongguk.ac.kr
}

\begin{abstract}
Indoor location based service (ILBS) is one of the current hottest research issues. ILBS cannot be realized without indoor positioning solutions. Among the many existing indoor positioning methods, the Wireless Local Area Network (WLAN) based trilateration is one of the most economic and easiest methods to be installed. The WLAN-based trilateration estimates the distance between an access point (AP) and a mobile device with the signal strength of the signal from the AP. Then, the WLAN-based trilateration estimates the location of the mobile device with the estimated distances and the coordinates of the APs. The first contribution of this paper is to propose a floor map repository that allows users to upload floor map files (AutoCAD files) and register coordinates of APs so that any software developer can use them after getting authorization. One of the drawbacks of the trilateration is its inaccuracy. The RF (radio frequency) signals between APs and a mobile device are very sensitive to the noise and the estimated distances are very inaccurate. The second contribution of this paper is that after discussing the pros and cons of various modified versions of trilateration, we find our own solution for indoor positioning and implement and publish our solution as a web service so that any software developer can use it.
\end{abstract}

Keywords: Indoor Positioning; Trilateration; WLAN; Access Point; RSSI

\section{Introduction}

Location based services (LBS) are information services accessible with mobile devices through the mobile network and utilizing the ability to make use of the location of the mobile device [1]. LBS services include identifying a location of a person or object, parcel tracking, vehicle tracking, mobile commerce, weather service, game, traffic advisories, and so on. Since these LBS services are so helpful to people's daily life, so many scientists and engineers are developing new technologies and applications of LBS every day.

Since LBS cannot be realized unless the location of the mobile device is identified, positioning is one of the essential techniques in LBS development. For outdoor LBS, Global Positioning System (GPS) provides general solution for positioning. However, GPS signal is so weak indoor or underground that we cannot determine user's location with GPS alone. Consequently, techniques for indoor positioning have been studied by many researchers. Active Badge [2], Active Bat [3] and Cricket [4] are pioneers of indoor positioning. These systems are highly accurate. But they require special equipments dedicated for positioning [5].

Many indoor positioning systems which do not require special equipments have also been developed. Most of them utilize WLAN (Wireless Local Area Network) equipments. 
Nowadays, WLAN is being serviced everywhere including college campuses, airports, hotels and even homes [5]. There are many alternative techniques we can choose in implementing WLAN based indoor positioning. Among them, the trilateration is one of the most economic and easiest one to be installed.

The trilateration estimates the distance between an access point (AP) and a mobile device with the radio frequency (RF) propagation loss model which is a simple mathematical expression representing the relationship between the received signal strength (RSS) and the distance between the sender and the receiver. Then, it estimates the location of the mobile device with the estimated distances and the coordinates of the APs. That is, in order for the trilateration to be applied, the coordinates of the APs must be available. Therefore, one of the main purposes of this paper is to propose a repository of floor maps and coordinates of points of interest (POI) including APs so that any software developer can use them after getting authorization.

Web services are applications that perform desired tasks. Service composition is the construction of complex services to enable different tasks. The author of [6] introduced a new algorithm for effective construction of the primitive services according to their quality criteria. The authors of [7] proposed ontological technique to apply user level quality of service that provides two different levels to serve Web service with proper quality by contribution value. An inspection of recent research achievements related to Service Oriented Architecture (SOA) testing is presented in [8]. Further the ways to improve functional testing of SOA applications created using Web services is explored. The authors of [9] showed the convergence model for NGN based Web Services and provides a detailed scenario of each convergence model in form of Web Services. The authors of [10] presented an experimental evaluation of the performance of different SOAP variants: standard SOAP, Swa/MIME, and SOAP/MTOM.

RSS is influenced by so many environmental parameters and establishing an appropriate RF propagation loss model is very difficult. As the result, one of the drawbacks of the trilateration is that it is less accurate than other methods and it sometimes returns an inordinately wrong answer. Therefore, we investigate the pros and cons of various modified versions of trilateration and propose our own solution for indoor positioning.

\section{Versions of Trilateration}

\subsection{Trilateration}

If we measure $N$ ranges, $r_{1}, r_{2}, \ldots, r_{N}$ from $N$ base stations, $n_{1}=\left(X_{1} Y_{1} Z_{1}\right)^{T}, \ldots, n_{N}=\left(X_{N} Y_{N} Z_{N}\right)^{T}$ to a mobile terminal, $m=(x y z)^{T}$ as shown in Figure 1, then we can estimate the coordinates of $m$ by using trilateration. By squaring, we can obtain the following expression for $r_{i}^{2}$ :

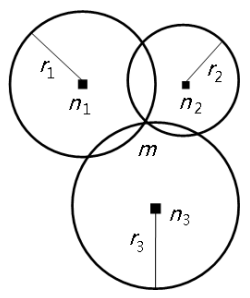

Figure 1. A diagram to illustrate trilateration 
$\left(x-X_{i}\right)^{2}+\left(y-Y_{i}\right)^{2}+\left(z-Z_{i}\right)^{2}=r_{i}^{2},($ for $i=1,2, \ldots, N)$

By subtracting $r_{1}^{2}$ from $r_{i}^{2}(i=2, \ldots, N)$, we have $A \vec{x}=\vec{b}$, where

$$
\begin{gathered}
A=2\left[\begin{array}{ccc}
\left(X_{2}-X_{1}\right) & \left(Y_{2}-Y_{1}\right) & \left(Z_{2}-Z_{1}\right) \\
\vdots & \vdots & \vdots \\
\left(X_{N}-X_{1}\right) & \left(Y_{N}-Y_{1}\right) & \left(Z_{N}-Z_{1}\right)
\end{array}\right], \quad \vec{x}=\left[\begin{array}{c}
x \\
y \\
z
\end{array}\right] \\
\vec{b}=\left[\begin{array}{c}
\left(X_{2}^{2}-X_{1}^{2}\right)+\left(Y_{2}^{2}-Y_{1}^{2}\right)+\left(Z_{2}^{2}-Z_{1}^{2}\right)-\left(r_{2}^{2}-r_{1}^{2}\right) \\
\vdots \\
\left(X_{N}^{2}-X_{1}^{2}\right)+\left(Y_{N}^{2}-Y_{1}^{2}\right)+\left(Z_{N}^{2}-Z_{1}^{2}\right)-\left(r_{N}^{2}-r_{1}^{2}\right)
\end{array}\right]
\end{gathered}
$$

When the coordinates are 3 dimensional, we need to have at least 4 base stations. Applying the MMSE (Minimum Mean Square Error) method, we can estimate the location of $m, \hat{\vec{x}}$ with the following position estimates[11]:

$$
\hat{\vec{x}}=\left(A^{T} A\right)^{-1} A^{T} \vec{b}
$$

\subsection{Kalman filter}

The author of [12] proposed a new algorithm which is very similar to the Kalman filter process. It is described as follows:

$$
\begin{aligned}
& \Delta=\left(B^{T} B\right)^{-1} B^{T} F=\left[\frac{\Delta x}{\Delta y}\right] \quad, \text { where } \\
& B=\left[\begin{array}{ll}
\frac{\left(x_{1}-x_{e}\right)}{\sqrt{\left(x_{1}-x_{e}\right)^{2}+\left(y_{1}-y_{e}\right)^{2}}} & \frac{\left(y_{1}-y_{e}\right)}{\sqrt{\left(x_{1}-x_{e}\right)^{2}+\left(y_{1}-y_{e}\right)^{2}}} \\
\frac{\left(x_{i}-x_{e}\right)}{\sqrt{\left(x_{i}-x_{e}\right)^{2}+\left(y_{i}-y_{e}\right)^{2}}} & \frac{\left(y_{i}-y_{e}\right)}{\sqrt{\left(x_{i}-x_{e}\right)^{2}+\left(y_{i}-y_{e}\right)^{2}}}
\end{array}\right] \quad \text { and } \\
& F=-\left[\begin{array}{l}
d_{1}-\sqrt{\left(x_{1}-x_{e}\right)^{2}+\left(y_{1}-y_{e}\right)^{2}} \\
\ldots \\
d_{i}-\sqrt{\left(x_{i}-x_{e}\right)^{2}+\left(y_{i}-y_{e}\right)^{2}}
\end{array}\right]
\end{aligned}
$$

where, $\left(x_{i}, y_{i}\right)$ is $A P_{i}$ 's coordinates and $\left(x_{e}, y_{e}\right)$ is the current location of the mobile device. We can initialize $\left(x_{e}, y_{e}\right)$ with any value. With $\Delta$, we update the mobile user's position $\left(x_{e}, y_{e}\right)$ as follows:

$$
x_{e}=x_{e}+\Delta x, y_{e}=y_{e}+\Delta y \text {. }
$$




\subsection{Weighted Centroid Localization}

In [13], Weighted Centroid Localization (WCL) is described. WCL belongs to the category of coarse grained approaches. Considering Figure 1, WCL estimates the coordinates of $m$ as follows:

$$
\hat{x}=\frac{\sum_{i=1}^{N} \frac{x_{i}}{r^{i}}}{\sum_{i=1}^{N} \frac{1}{r^{i}}} \quad \hat{y}=\frac{\sum_{i=1}^{N} \frac{y_{i}}{r^{i}}}{\sum_{i=1}^{N} \frac{1}{r^{i}}}
$$

\subsection{Our Method}

WCL has a significant shortcoming. That is, if the mobile terminal locates outside of the region determined by the lines connecting APs, then the estimation has a huge error. Moreover, the configuration of the region determined by the lines connecting the APs should meet some conditions. For example, suppose the mobile terminal receives signals from three APs and their coordinates are $(50,0),(0,100)$, and $(100,100)$ as shown in Figure 2. Suppose further that the signal strengths from the APs are all the same. Then, the mobile terminal's current location should be $(50,50)$, but the result of WCL is $(50,70)$. Considering this shortcoming, we propose our method.

We propose our version of trilateration that can be applied under the following assumptions:

1) We know the room where the mobile device is located

2) Rooms are square

3) APs are located at the four corners of a room

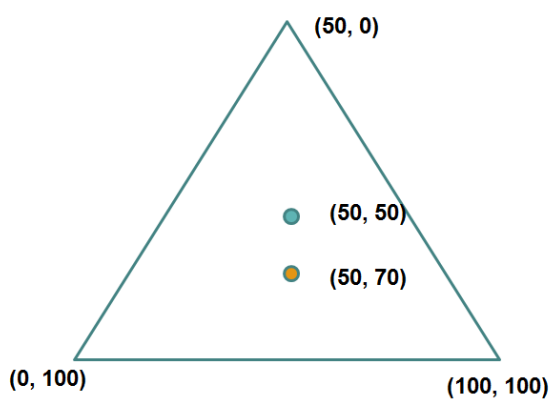

Figure 2. The result of WCL

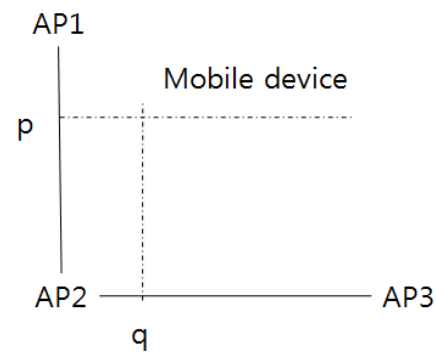

Figure 3. A diagram to explain our method 
Under the assumptions, we find $\mathrm{p}$ as follows:

$$
\left(\frac{r_{1} x_{2}+r_{2} x_{1}}{r_{1}+r_{2}}, \frac{r_{1} y_{2}+r_{2} y_{1}}{r_{1}+r_{2}}\right)
$$

where $\left(x_{i}, y_{i}\right)$ is $A P_{i}$ 's coordinates and $r_{i}$ is the distance between $A P_{i}$ and the mobile device. In the same manner, we find q. After that, we find the line perpendicular to the line (AP1, AP2) starting at p. We do the same for $\mathrm{q}$. Then the intersection of those lines is the location of the mobile device as is shown in Figure 3.

\section{AP Location Upload}

As LBS systems use the global map as their user interfaces, an ILBS most likely uses a floor map as its user interface. In order to apply the trilateration, an ILBS has to know the coordinates of APs. If any floor map and the coordinates of any AP are available anywhere, we can implement an ILBS system which runs wherever the user is. One of the two main purposes of this paper is to propose a repository system which can provide all the floor maps and the coordinates of all APs.

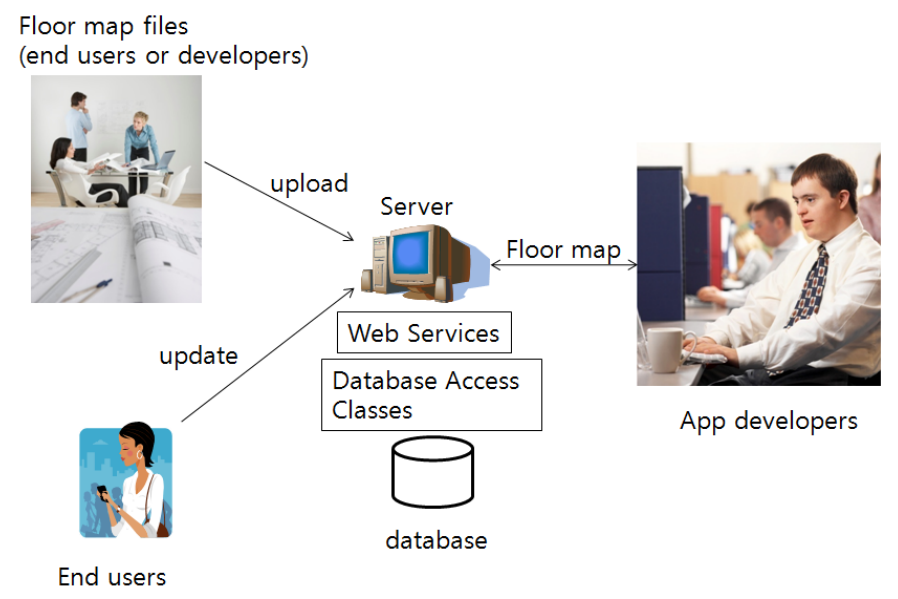

Figure 4.A conceptual model of the repository system

A conceptual model of our repository system is shown in Figure 4. We are collecting and saving all the floor maps in the world in the database. In order to collect all the floor maps and the coordinates, the repository system allows users to upload and update their own floor maps and the coordinates of the APs in their buildings. The system also allows the user to search a floor map, to use the image of selected floor map. It also allows the administrators to delete and update floor maps.

We built a prototype of the repository system. We started with implementing the following web services: upload a floor map, search a floor map, rendering a floor map, delete a floor map, upload the coordinates of an AP. 


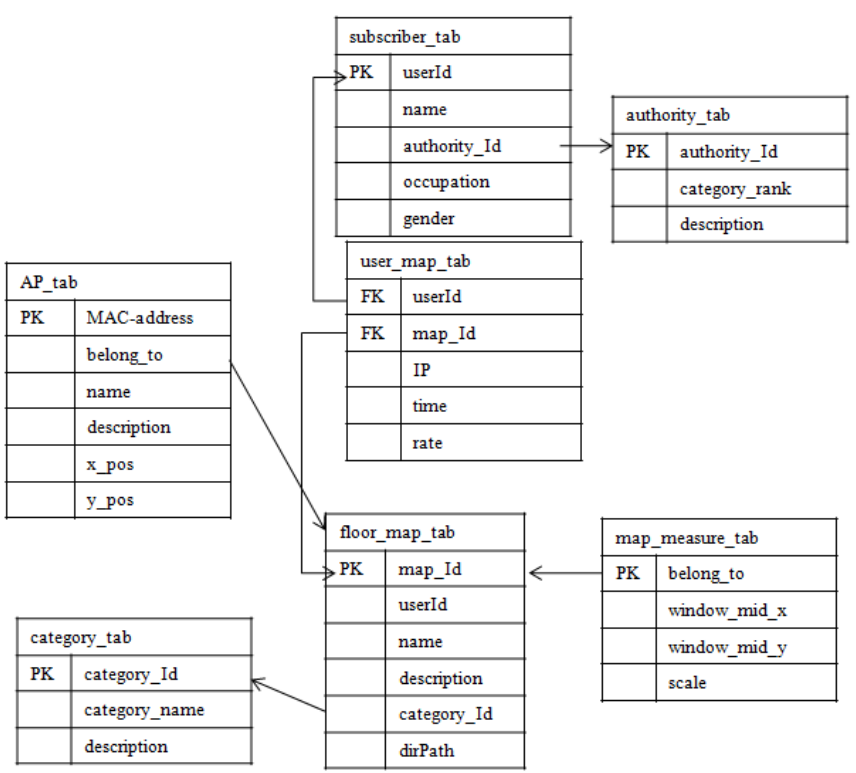

Figure 5.The structure of our database system

For the repository, we need a database (DB) system consisting of the tables shown in Figure 5. Information of floor maps is recorded in floor_map_tab. The userId represents the person who uploaded the floor map. Information of "who (userId) used which (map_Id) floor map" is recorded in user_map_tab. Once a user used a map, the user can evaluate the quality of the map.

\begin{tabular}{|c|c|}
\hline DrawingDao & DBConnector \\
\hline -DBConnector & -driver \\
\hline \multirow{4}{*}{$\begin{array}{l}\text { +DrawingDao0 } \\
\text { +registerDrawing0 } \\
\text { +re gisterDrawingMeasure() } \\
\text { + registerPOI0 } \\
\text { + deleteDrawing0 } \\
\text { + deleteDrawingMeasure } 0 \\
\text { +getAllDrawingName } 0 \\
\text { +getDrawingDirPath } 0 \\
\text { +getScale } 0\end{array}$} & \\
\hline & $\begin{array}{l}\text {-pwd } \\
\text {-conn }\end{array}$ \\
\hline & +getConnection 0 \\
\hline & \\
\hline
\end{tabular}

Figure 6. The structure of the classes for DB manipulation

The coordinates of APs are recorded in AP_tab. An AP is uniquely identified by its MAC address. The fields of $\mathrm{x} \_$pos and y_pos represent the coordinates of the AP corresponding to MAC_address.

One of the most important functions of the repository is providing images of floor maps which are AutoCAD files. This implies that the repository system has to draw an image for a given AutoCAD file. Since the image is to be rendered on a smart phone, the size of the image is usually much smaller than the size of the AutoCAD image. The ratio of the size of the image for smart phone to AutoCAD image size is recorded in scale field of map_measure_tab.

Our database system provides DBConnector class and DrawingDao class. In the former, getConnection() method for DB connection is implemented. In the latter, many 
methods of manipulating DB are defined. The structure of these classes is shown in Figure 6.

The repository system is basically a client-server system. The database system and the web services are parts of the server. One of the most typical clients of this system would be a smartphone app. We have implemented an Android app that allows the user upload AutoCAD files, displays the image of the requested floor map, and allows the user upload the coordinates of POIs. If a user touches the point on the floor map, then the app converts the coordinates of the window coordinates of the point into the coordinates of the floor map. One of the most tricky parts of this app is to find the image coordinate of the touched point. This app renders the image of a floor map on an android:scaleType ImageView and defines the scaleType as MATRIX so that we can use Matrix class in order to move, zoom, and skew the image. Matrix class has many methods of manipulating the image. For example, postScale method of Matrix class zooms the image. Matrix class has $3 \times 3$ matrix and the attributes of the image are recorded in this matrix. Using this information we can convert screen coordinate $(\mathrm{x}, \mathrm{y})$ into image coordinate as follows:

float[] values = new float[9]; matrix.getValues(values); image $\mathrm{x}=\mathrm{x} /$ values[0] - values[2]/values[0]; image $y=y /$ values[4] - values[5]/values[4];

\section{Implementation and Experiments}

Using the web services discussed in Section 3, we have implemented a mobile app with which a user can upload an AutoCAD file (floor map) and location information of an AP. The following figures in Figure 7 are screenshots of the client part of our repository system running on a smartphone.

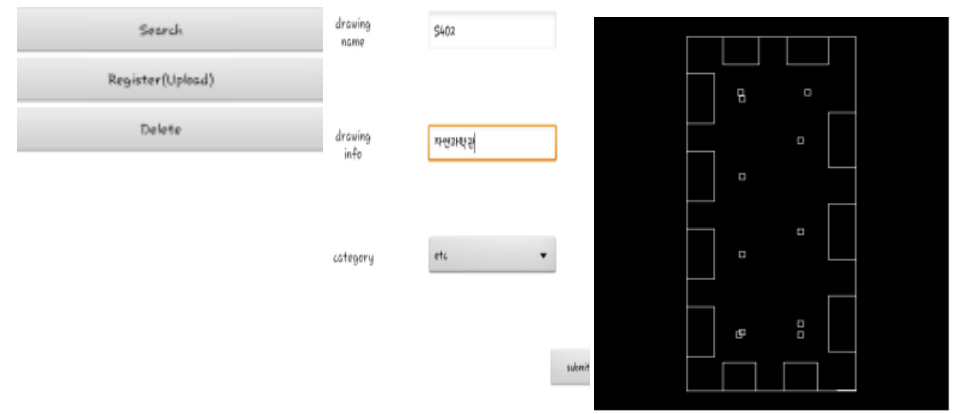

(a) The main page (b) The upload page (c) The display page

Figure 7. Screenshots of the repository mobile app running on a smartphone

Since the image of the floor map is displayed on an ImageView defined as android:scaleType="matrix", it can be zoomed and moved and the information of image manipulation is recorded in a matrix.

We have also implemented and tested those versions of the trilateration discussed in Section 2 as web services. Our test results showed that the Trilateration version 2 does not work properly. Therefore, we changed it with the extended Kalman filter [5, 6]. The web services we have implemented are shown in Figure 8. 


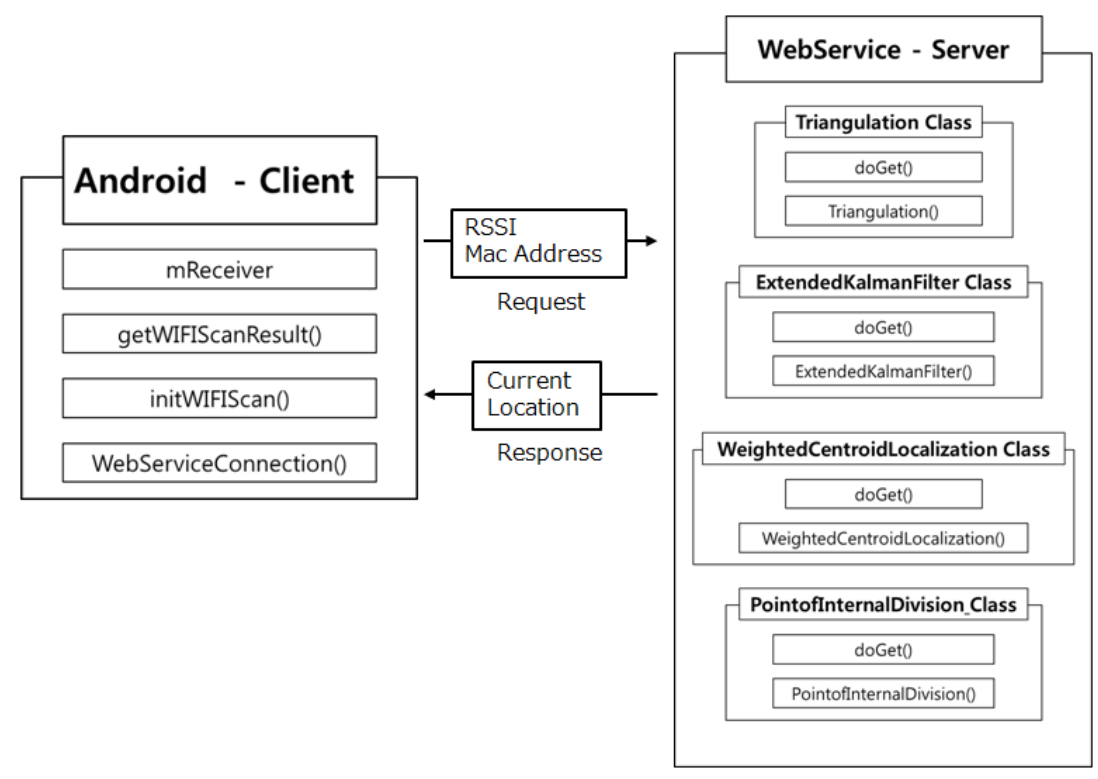

Figure 8. The structure of our indoor positioning system

We have performed experiments of testing those web services in a square-shaped room where the APs are displaced at the four corners. In this experiments we have run each of the versions 100 times. The test results showed that the average error of version 4 is $2.45 \mathrm{~m}$ whereas the average error of version 1 is $10.95 \mathrm{~m}$ and that of version 3 is $3.12 \mathrm{~m}$. The extended Kalman filter was converged after 20 repetitions and the error of the converged point was $2.57 \mathrm{~m}$.

\section{The Proposed Indoor Positioning}

From our experiments we found that the trilateration version 4 is most accurate if the assumptions are met. The Kalman filter is also accurate if it is applied on a sequence of 20 tuples tup ${ }_{i}$ where tup ${ }_{i}$ consists of many RSSIs (Received Signal Strength Indicators) collected at time $i$. Therefore, it takes a long time and consumes a lot of battery power to collect many tuples and execute the Kalman filter process that many times. If it is used to trace locations of the mobile device, it has to execute the process periodically, every second for example, and consumes a lot of computing resources and battery power. Thus, we propose indoor positioning process as shown in Figure 9. It scans WLAN card to collect RSSIs and MAC addresses of APs. Then, it retrieves the coordinates of those APs. If the situation satisfies the assumptions made by the trilateration version 4 , then we apply that version and return the result as the location of the mobile smartphone. Otherwise, if the battery power level is high then we apply the Kalman filer. If neither those assumptions are met nor the battery power level is high then we apply version 3. 


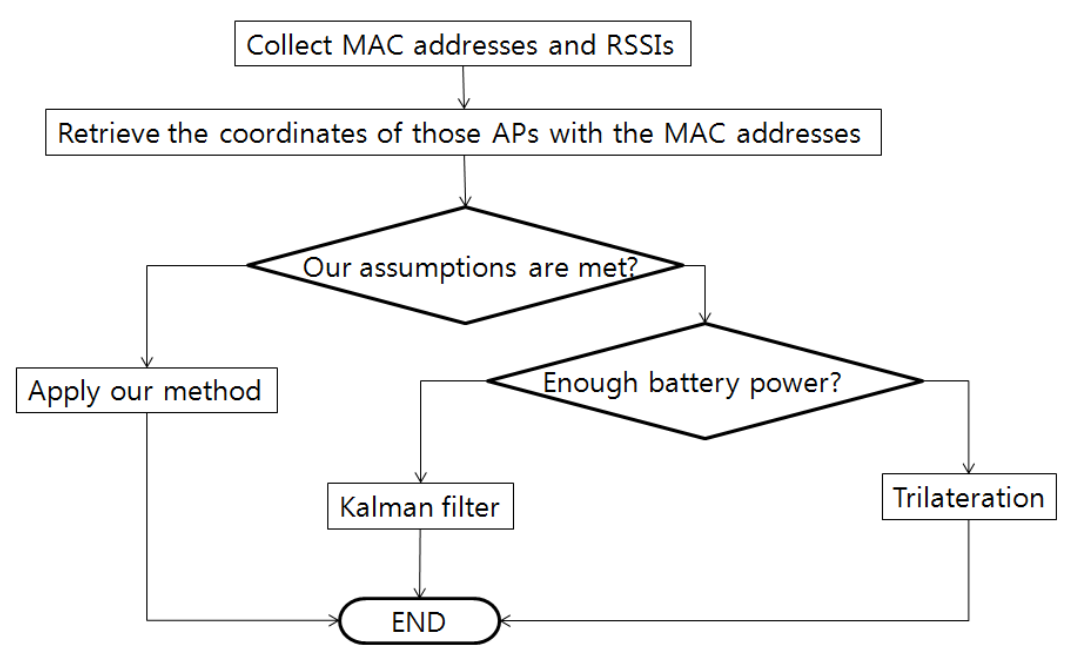

Figure 9. Description of our indoor positioning process

\section{Implementation}

We have implemented those positioning algorithms discussed in the paper as shown in Figure 10. The name of the Web service reflects the algorithm it performs. For example, WeightedCentroidLocalization is the Web service that performs the method discussed in Section 2.3 Weighted Centroid Localization. PointofInternalDivision is the Web service that performs our algorithm discussed in Section 2.4.

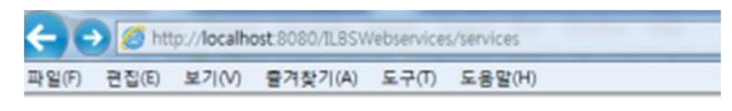

\section{And now... Some Services}

- WeightedCentroidLocalization (wsdl)

- Trilateration (wsdl)

- PointoflnternalDivision (wsdl)

- ExtendedKalmanFilter (wsdl)

- AdminService (wsdl) - AdminService

- FSMIndoorPositioning (wsdl)

\section{Figure 10. Web services for indoor positioning}

We have implemented Web services for the floor map repository system as shown in Figure 11. In AccessPoint.Java/Drawing.Java, the Web services that create, retrieve, update, and delete the coordinates of an AP/a floor map are defined. 


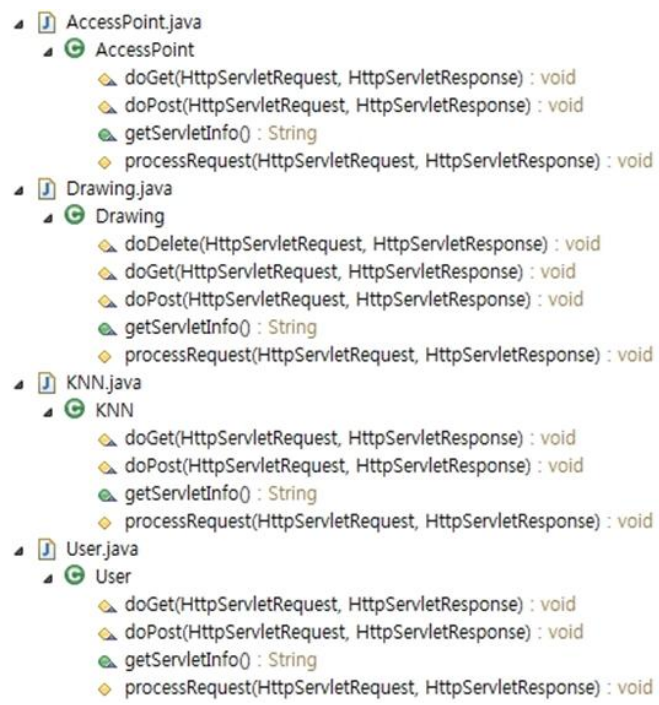

Figure 11. Web services for the floor map repository system

\section{Conclusions}

The WLAN-based trilateration is one of the most economic and easiest to implement indoor positioning methods. In order for the trilateration to be executed on a smartphone, the coordinates of the APs around the smartphone should be available. This paper proposed the repository system that provides all the coordinates of all the APs in the world. The system provides web services for uploading, deleting, and retrieving floor maps and coordinates of APs to/from the repository system so that any software developer can use them.

The trilateration suffers from the noise sensitiveness of RF signal and relatively inaccurate. Sometimes, the coordinates returned by the trilateration are inordinately wrong. After discussing the pros and cons of various versions of trilateration, we have proposed our own indoor positioning process based on them. We implemented our indoor positioning process as a web service so that any software developer can use it.

If there were not Google Maps or GPS, LBS software were not so abundant today. Our repository of floor maps and coordinates of APs is to ILBS (Indoor LBS) as Google Maps is to LBS. The web service of our positioning process is to ILBS as GPS is to LBS. To the best knowledge of ours, this paper is the first attempt to provide a universal solution for indoor positioning and user interface of ILBS system. Therefore, the repository system and the web services this paper implemented and published will significantly contribute to the growth of the ILBS industry.

\section{Acknowledgments}

This research was supported by Basic Science Research Program through the National Research Foundation of Korea(NRF) funded by the Ministry of Education(NRF-2011-0006942) and by 'Development of Global Culture and Tourism IPTV Broadcasting Station' Project through the Industrial Infrastructure Program for Fundamental Technologies funded by the Ministry of Knowledge Economy (10037393). 


\section{References}

[1] M. H. Khairullah and R. H. Banna, "BlueAd: A Location based Service using Bluetooth", International Journal of Computer Applications (0975 - 8887), vol. 43, no. 15, (2012) April, pp. 19-22.

[2] R. Want, A. Hopper, V. Falcao and J. Gibbons, "The active badge location system", ACM Transactions on Information Systems, vol. 10, no. 1, (1992), pp. 91-102.

[3] A. Harter and A. Hopper, "A new location technique for the active office", IEEE Personal Communications, vol. 4, no. 5, (1997), pp. 43-47.

[4] N. Priyanthat, A. Chakraborty and H. Balakrishnan, "The Cricket location-support system", Proceedings of 6th ACM International Conference on Mobile Computing and Networking, Boston, MA, (2000).

[5] J. Yim and S. Jeong, "Improvement of Kalman Filters for WLAN Based Indoor Tracking", Expert Systems With Applications, vol. 37, no. 1, (2010) January, pp. 426-433.

[6] M. Bazarganigilani, "Web Service Selection Using Quality Criteria and Trust Based Routing Protocol", IJSIA, vol. 6, no. 4, (2012) October, pp. 109-118.

[7] Y. Ha and H. -S. Park, "QoS based Client information for semantic Web service", IJSEIA, vol. 3, no.1, January (2009), pp. 61-68

[8] P. Kalamegam and Z. Godandapani, "A Survey on Testing SOA Built using Web Services", IJSEIA, vol. 6, no. 4, (2012) October, pp. 91-104.

[9] Y. Lee, K. Lee and S. Lee, "Web Services Deployment Model Based on WSG (Web Services Gateway) in NGN", IJSEIA, vol. 7, no. 1, (2013) January, pp. 229-236.

[10] S. Cha, Y. Hwang, Y. Chang, K. Kim and K. Lee, "Design and Evaluation of Experiment Methods for Improving Performance In GIS Web Services”, IJMUE, vol. 3, no. 1, (2008) January, pp. 27-44.

[11] J. Yim, C. Park, J. Joo and S. Jeong, "Extended Kalman Filter for Wireless LAN Based Indoor Positioning", Decision Support Systems, vol. 45, (2008), pp. 960-971.

[12] N. Verma, "Determining the Algorithm for Location Based Services Using Indoor Positioning Techniques, "International Journal of Advanced Research in Computer Science and Software Engineering, vol. 2, Issue 7, (2012) July, pp. 411-416.

[13] A. Lewandowski, S. Michaelis, C. Wietfeld, J. Klaue and M. Kubisch, "In-cabin localization solution for optimizing manufacturing and maintenance processes for civil aircrafts", Position Location and Navigation Symposium (PLANS), IEEE/ION, (2012), pp. 1257 - 1264.

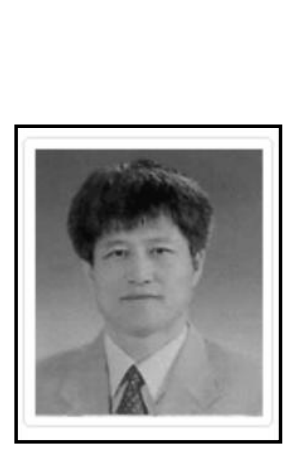

\section{Author}

\section{Jaegeol Yim}

Jaegeol Yimreceived the M.S. and Ph.D. degrees in Computer Science from the University of Illinois at Chicago, in 1987 and 1990, respectively. He isa Professor in the Department of Computer Science at Dongguk University at Gyeongju Korea. His current research interests include Petri net theory and its applications, Location Based Service, AI systems, and multimedia systems.He has published more than 50 journal papers, 100 conference papers (mostly written in Korean Language), and several undergraduate textbooks. 
International Journal of Control and Automation

Vol.7, No.3 (2014) 